\title{
Universality in Molecular Halo Clusters
}

\author{
P. Stipanović, ${ }^{1}$ L. Vranješ Markić, ${ }^{1,2 *}$ I. Bešlić, ${ }^{1,3}$ and J. Boronat ${ }^{3}$ \\ ${ }^{1}$ Faculty of Science, University of Split, HR-21000 Split, Croatia \\ ${ }^{2}$ Department of Physics and Astronomy, University of Delaware, Newark, Delaware 19716-2593, USA \\ ${ }^{3}$ Departament de Física i Enginyeria Nuclear, Campus Nord B4-B5, Universitat Politècnica de Catalunya, \\ E-08034 Barcelona, Spain
}

(Received 15 August 2014; published 16 December 2014)

\begin{abstract}
The ground state of weakly bound dimers and trimers with a radius extending well into the classically forbidden region is explored, with the goal to test the predicted universality of quantum halo states. The focus of the study is molecules consisting of $\mathrm{T} \downarrow, \mathrm{D} \downarrow,{ }^{3} \mathrm{He},{ }^{4} \mathrm{He}$, and alkali atoms, where the interaction between particles is much better known than in the case of nuclei, which are traditional examples of quantum halos. The study of realistic systems is supplemented by model calculations in order to analyze how low-energy properties depend on the interaction potential. The use of variational and diffusion Monte Carlo methods enabled a very precise calculation of both the size and binding energy of the trimers. In the quantum halo regime, and for large values of scaled binding energies, all clusters follow almost the same universal line. As the scaled binding energy decreases, Borromean states separate from tango trimers.
\end{abstract}

DOI: 10.1103/PhysRevLett.113.253401

PACS numbers: 36.40.-c, 21.45.-v, 31.15.ac

Universality is important in nearly all areas of physics, since it enables the establishment of connections between phenomena at different energy and length scales. It is also a key characteristic of quantum halo states, usually defined as bound states which extend far into the classically forbidden regions [1,2]. They were first recognized $[3,4]$ and traditionally explored in nuclear physics $[1,2,5]$, but are also known to exist in molecular physics and have been recently created in ultracold gases using Feshbach resonances [6]. Known halo dimers extend over an energy scale of 16 orders of magnitude. Universality means that the details of the potential do not matter, rather all properties of dimers can be expressed in terms of the scattering length $a$.

The concept of universality and quantum halo states was extended to systems with more particles $[1,7,8]$. As in the case of dimers, if universality exists the properties of the system are describable by any interparticle potential where one or a few scattering parameters are the same. It became clear very soon that the radial extension of the cluster is a fundamental quantity which can be used to characterize the states. In order to analyze systems across different physics fields, dimensionless scaling variables were introduced and scaled size and scaled energy compared $[9,10]$. The study of Jensen et al. suggests that scaling of trimers is approximately universal [10]. Universality is expected also in excited Efimov states [10]. However, such comparisons included mostly models of nuclear systems which are assumed to be separable into a structureless core and one or more halo particles. Realistic molecular systems are lacking, but, at the same time, interactions between atoms in weakly bound clusters are much better known than in nuclear systems. Thus, molecular systems can be regarded as a bridge between nuclear halo states and halo states which appear in ultracold gases. In this Letter, we show that molecular clusters are the best suited systems for testing the universality of scaling between the energy and size of quantum halo clusters.

In 2005 Jensen et al. [1] predicted a number of molecular systems which could be candidates for quantum halo states. However, at that time data for both the energy and size of the clusters were available for only ${ }^{4} \mathrm{He}_{2},{ }^{4} \mathrm{He}_{2}{ }^{3} \mathrm{He}$, and the excited state of ${ }^{4} \mathrm{He}_{3}$, whose quantum halo character was thus confirmed. In our previous work on small clusters of helium and spin-polarized tritium ( $\mathrm{T} \downarrow$ ) we also predicted a number of possible quantum halo clusters [11]. Several studies of the $(\mathrm{T} \downarrow)_{3}$ and He-alkali dimers and trimers revealed a weak binding of some of these systems as well [12-20].

Experimentally, several molecular quantum halos have been detected so far. Among them, using diffraction from the nanoscale grating, the ${ }^{4} \mathrm{He}_{2}$ dimer [21] and the ${ }^{4} \mathrm{He}_{2}{ }^{3} \mathrm{He}$ trimer [22]. Recently, a He-Li dimer has been detected as well [23], offering hope that molecular halo systems with more than two particles could be observed and their properties measured.

In this work, we study the ground state of selected molecular dimers and trimers, that are candidates for quantum halo states due to their small binding energy, with the goal of testing the universality of the predicted scaling laws [9].

In order to introduce the scaling variables we start the discussion with the dimers. One has to introduce a length scale $R$ with which to compare the size of the dimer, usually quantified through the root-mean-square radius, $\sqrt{\left\langle r^{2}\right\rangle}$, with $r$ the distance between the particles. In the first model, $R$ is identified with the outer classical turning point [24]. 
In this case, one can define a quantum halo as a two-body system with a probability to be in a classically forbidden region higher than $50 \%$, or as is commonly stated $\left\langle r^{2}\right\rangle / R^{2}>2$. The other variable is the binding energy, in the scaled form $\mu B R^{2} / \hbar^{2}$, where $B$ equals the absolute value of the ground-state energy and $\mu$ is the reduced mass of the dimer. We numerically solved the Schrödinger equation for $\mathrm{He}-\mathrm{He}$, He-alkali, and He-alkaline-earth systems, using several interaction potentials [15,25-30]. The results for the realistic dimers are presented in Fig. 1 as points, while the line corresponds to the fit through squarewell (SQW) model calculations. Our results are in agreement with the published values of dimer energies from other authors [15,31-33], while the prediction of $\left\langle r^{2}\right\rangle$ is usually not given. The most notable example of a molecular halo dimer is ${ }^{4} \mathrm{He}_{2}$. Different models for He-He interactions give binding energies from $-1.88 \mathrm{mK}$ in the case of the Symmetry-Adapted Perturbation Theory potential [27] to $-1.29 \mathrm{mK}$ for the Tang-Toennies-Yiu potential [26]. Further examples of halo dimers are the He-alkali systems, the most extended being ${ }^{4} \mathrm{He}-{ }^{6} \mathrm{Li}$. All studied dimers follow the same curve, even slightly below the quantum-halo limit, indicated by the horizontal line. As the scaled energy is even more increased, the SQW model clearly differs from the realistic molecular clusters, represented in this energy range by He-alkaline-earth dimers.

Since this definition of scaling size $R$ cannot be straightforwardly extended to systems of more particles, Fedorov et al. [34] proposed to define $R$ as the radius of the equivalent SQW (ESQW) potential, which has the same

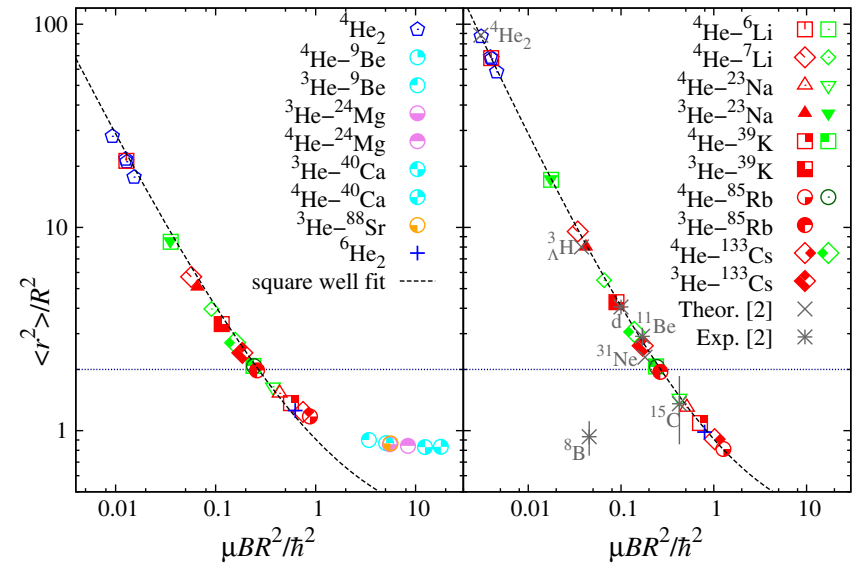

FIG. 1 (color online). Size vs ground-state energy scaling plot for molecular two-body halos compared with the nuclei data [2]. On the left (right) plot $R$ is determined as the outer turning point (the width of the equivalent SQW). The horizontal line is the quantum halo limit and the dashed one the fit through SQW model results. Labels are valid for both sides of the plot. For ${ }^{4} \mathrm{He}_{2}$ the symbols from left to right correspond to interactions from Refs. [26,25,29] and [27]. For He-alkali dimers, the left (right) symbol corresponds to interaction from Ref. [28] (Ref. [15]). The size of the symbols is larger than the error bar. $s$-wave scattering length and effective range as the original potential. We determined the scattering length and effective range for all $\mathrm{He}-\mathrm{He}$ and He-alkali studied systems and, from them, we built the ESQW potentials. Our results are presented in the right-hand side plot of Fig. 1, where the results with real and ESQW potentials are equal. The scaled size of quantum halos is somewhat larger in this case. All of the molecular dimers lie on the line fitted through the SQW models: ESQW and modified SQW (MSQW) models. The latter have the mass of molecular clusters, but modified depth and width with respect to the ESQW. Notably, this holds even below the quantum halo limit $\left\langle r^{2}\right\rangle / R^{2}=2$. The values for the nuclei taken from Ref. [2] in most cases also follow the universal line. The exception is ${ }^{8} \mathrm{~B}$, outside the halo region, where the centrifugal and Coulomb barrier due to its core $+p$ nature presumably reduce its size [2].

The second definition of the scaling radius was extended to trimers [1]. The size of the system is measured by the root-mean-square hyperradius $\sqrt{\left\langle\rho^{2}\right\rangle}, \rho$ given by

$$
m \rho^{2}=\frac{1}{M} \sum_{i<k} m_{i} m_{k}\left(\mathbf{r}_{i}-\mathbf{r}_{k}\right)^{2},
$$

where $m$ is an arbitrary mass unit, $m_{i}$ the particle mass of species $i$, and $M$ the total mass of the system. Generalizing the hyperradius (1), Jensen et al. [1] defined the size scaling parameter $\rho_{0}$ as

$$
m \rho_{0}^{2}=\frac{1}{M} \sum_{i<k} m_{i} m_{k} R_{i k}^{2}
$$

where $R_{i k}$ is the two-body scaling length of the $i-k$ system, which is calculated as the width of the ESQW potential between the $i$ and $k$ species. This definition [Eq. (2)] enabled the comparison to two-body halos and the analogous definition of the quantum halo as $\left\langle\rho^{2}\right\rangle / \rho_{0}^{2}>2$.

In order to test the universality of the quantum halos a very accurate calculation of the energy and size of these extremely extended clusters needs to be done. Although demanding for weakly bound trimers, this goal can be achieved using the diffusion Monte Carlo (DMC) method [35] with pure estimators [36]. The DMC method solves, within a stochastic approach, the Schrödinger equation written in imaginary time. For long simulation times, providing that the guiding wave function used for importance sampling has nonzero overlap with the exact groundstate wave function, the exact ground-state energy of a $N$-body bosonic system can be obtained (within some statistical uncertainty). We used guiding wave functions of the Jastrow form, constructed as a product of two-body correlation functions $F_{i j}(r), \quad \psi(\boldsymbol{R})=\prod_{i<j=1}^{n} F_{i j}\left(r_{i j}\right)$. For realistic potential models we chose 


$$
F_{i j}(r)=\frac{1}{r} \exp \left[-\left(\alpha_{i j} / r\right)^{\gamma_{i j}}-s_{i j} r\right]
$$

where $r$ is the interparticle distance, and $\alpha_{i j}, \gamma_{i j}$ and $s_{i j}$ are variational parameters. For the SQW model we used

$$
F_{i j}(r)= \begin{cases}\frac{\sin \left(k_{i j} r\right)}{r} & r \leq L_{i j} \\ \exp \left[\frac{k_{i j}\left(r-L_{i j}\right)}{\tan \left(k_{i j} L_{i j}\right)}\right] \frac{\sin \left(k_{i j} L_{i j}\right)}{r} & r>L_{i j}\end{cases}
$$

with variational parameters $k_{i j}$ and $L_{i j}$. Both in Eqs. (3) and (4), the parameters were optimized using the variational Monte Carlo (VMC) method.

Our results for trimers, obtained using interaction potentials $[15,25,28,37-39]$, are presented in Fig. 2 and a more detailed sample is presented in Ref. [40]. The empty or 3/4-empty symbols belong to the realistic systems, while the full or 3/4-full ones come from the MSQW models. Where a molecular system is represented by two equal symbols the left one comes from the real two-body potential, and the right one from the ESQW. Here, we see that these two points lie very close, on the same line, and in some cases they are the same within the error bars. In fact, universality in this context means that clusters can be described by any potential with a common scattering length and effective range. The results for the binding energy of trimers containing only isotopes of $\mathrm{He}$ and/or $\mathrm{H}$ are in good agreement with other published works, [12,13,31,41,42]. However, previous work [1], also given in Fig. 2 appears to

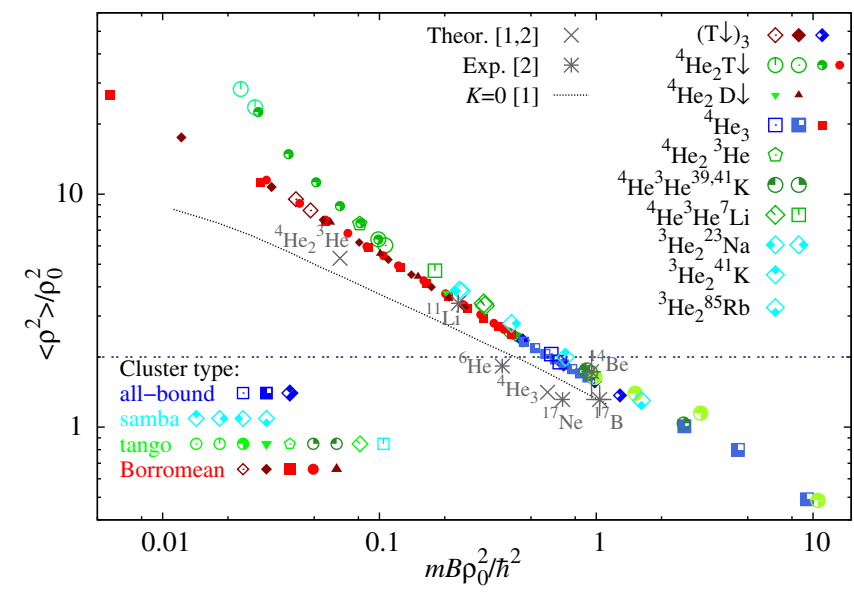

FIG. 2 (color online). Size vs energy scaling plot for groundstate molecular three-body halos. Empty or 3/4-empty symbols designate the realistic, and full or 3/4 full symbols the MSQW potentials. In the case of ${ }^{4} \mathrm{He}_{2} \mathrm{~T} \downarrow$, the left (right) symbol for realistic potential corresponds to He-T interaction from Ref. [38], (Ref. [39]). For He-alkali trimers, the left (right or only one) symbol corresponds to He-alkali interaction from Ref. [15] (Ref. [28]). Horizontal line represents the quantum halo limit. For comparison, we include the $K=0$ line from Ref. [1] and the results from Ref. [2]. The size of the symbols is larger than the error bars. underestimate the sizes of both ${ }^{4} \mathrm{He}_{3}$ and ${ }^{4} \mathrm{He}_{2}{ }^{3} \mathrm{He}$, placing them below the universal line. For the He-alkali trimers, and to the best of our knowledge, no results exist with the He-alkali interaction potential by Cvetko et al. [28], while other authors, who used the potential by Kleinekathöfer et al. [15], or its older version [43], modeled the He-He interaction with a weaker form than the HFD-B(He) [25] potential used in the present work. Thus, we predict somewhat stronger binding for ${ }^{3} \mathrm{He}^{4} \mathrm{He}^{7} \mathrm{Li}$ and ${ }^{3} \mathrm{He}_{2}{ }^{23} \mathrm{Na}$ than Yuan and Lin [16]. For ${ }^{3} \mathrm{He}^{4} \mathrm{He}^{39} \mathrm{~K}$ our DMC energy is between the lower and upper bounds predicted in Ref. [18], while for ${ }^{3} \mathrm{He}_{2}{ }^{85} \mathrm{Rb}$ we found a bound state only using the potential [28], contrary to the findings of Ref. [14].

Different types of trimer states, marked with symbols in Fig. 2, are possible: Borromean [1], where no two-body subsystem is bound; tango [44], where only one subsystem is bound; sambas [45], with two bound dimers; and allbound with three bound dimer subsystems. The only realistic molecular Borromean trimer (empty diamond) is $(\mathrm{T} \downarrow)_{3}$, confirming the predictions of Refs. [12,13,42,46]. We obtained other Borromean clusters using MSQW models of ${ }^{4} \mathrm{He}_{3},{ }^{4} \mathrm{He}_{2} \mathrm{D} \downarrow,{ }^{4} \mathrm{He}_{2} \mathrm{~T} \downarrow$ mass, with reduced $\mathrm{He}-\mathrm{He}$ and strengthened $\mathrm{He}-\mathrm{H}$ interaction potentials. All of these Borromean states have $\left\langle\rho^{2}\right\rangle / \rho_{0}^{2}>2$ and fall on the same line. Increasing the interaction strength, the Borromean line passes smoothly into the line of clusters which have all of their pairs bound. The only realistic cluster of this type we studied is ${ }^{4} \mathrm{He}_{3}$ (empty square), which is exactly on the border of quantum halo states. Other points represent clusters of ${ }^{4} \mathrm{He}_{3}$ or $(\mathrm{T} \downarrow)_{3}$ mass interacting with MSQW potentials.

Realistic tango clusters are ${ }^{4} \mathrm{He}_{2}{ }^{3} \mathrm{He}, \quad{ }^{4} \mathrm{He}_{2} \mathrm{~T} \downarrow$, ${ }^{4} \mathrm{He}^{3} \mathrm{He}^{7} \mathrm{Li},{ }^{4} \mathrm{He}^{3} \mathrm{He}^{39} \mathrm{~K}$, and ${ }^{4} \mathrm{He}^{3} \mathrm{He}^{41} \mathrm{~K}$, and all fall approximately on the same line. Not all tango states are quantum halos, and the criterion $\left\langle\rho^{2}\right\rangle / \rho_{0}^{2}>2$ includes ${ }^{4} \mathrm{He}_{2}{ }^{3} \mathrm{He},{ }^{4} \mathrm{He}_{2} \mathrm{~T} \downarrow$ and ${ }^{4} \mathrm{He}^{3} \mathrm{He}^{7} \mathrm{Li}$. Other tango states were again obtained using MSQW models. As the binding is reduced, the tango line separates from the Borromean line, in accordance with the prediction of Frederico et al. [8], obtained using renormalized zero-range two-body interactions. According to that work [8], for a given energy the size of the system increases going from Borromean, through tango, samba, and finally all bound states. Among studied clusters we did not find that samba or all-bound states separate from the joint Borromean and tango curve. However, it is not theoretically excluded that this separation would appear for significantly different mass compositions. Samba clusters ${ }^{3} \mathrm{He}_{2}{ }^{23} \mathrm{Na}$ and ${ }^{3} \mathrm{He}_{2}{ }^{41} \mathrm{~K}$ can be considered quantum halos.

Comparing our results with experimental values for nuclei, we find excellent agreement for ${ }^{11} \mathrm{Li}$ which is a Borromean state. Other nuclei fall below the $\left\langle\rho^{2}\right\rangle / \rho_{0}^{2}>2$ limit; however, ${ }^{14} \mathrm{Be}$ and ${ }^{17} \mathrm{~B}$ are within the error bar of the line formed by molecular clusters. ${ }^{6} \mathrm{He}$ (two neutrons in $p$ 

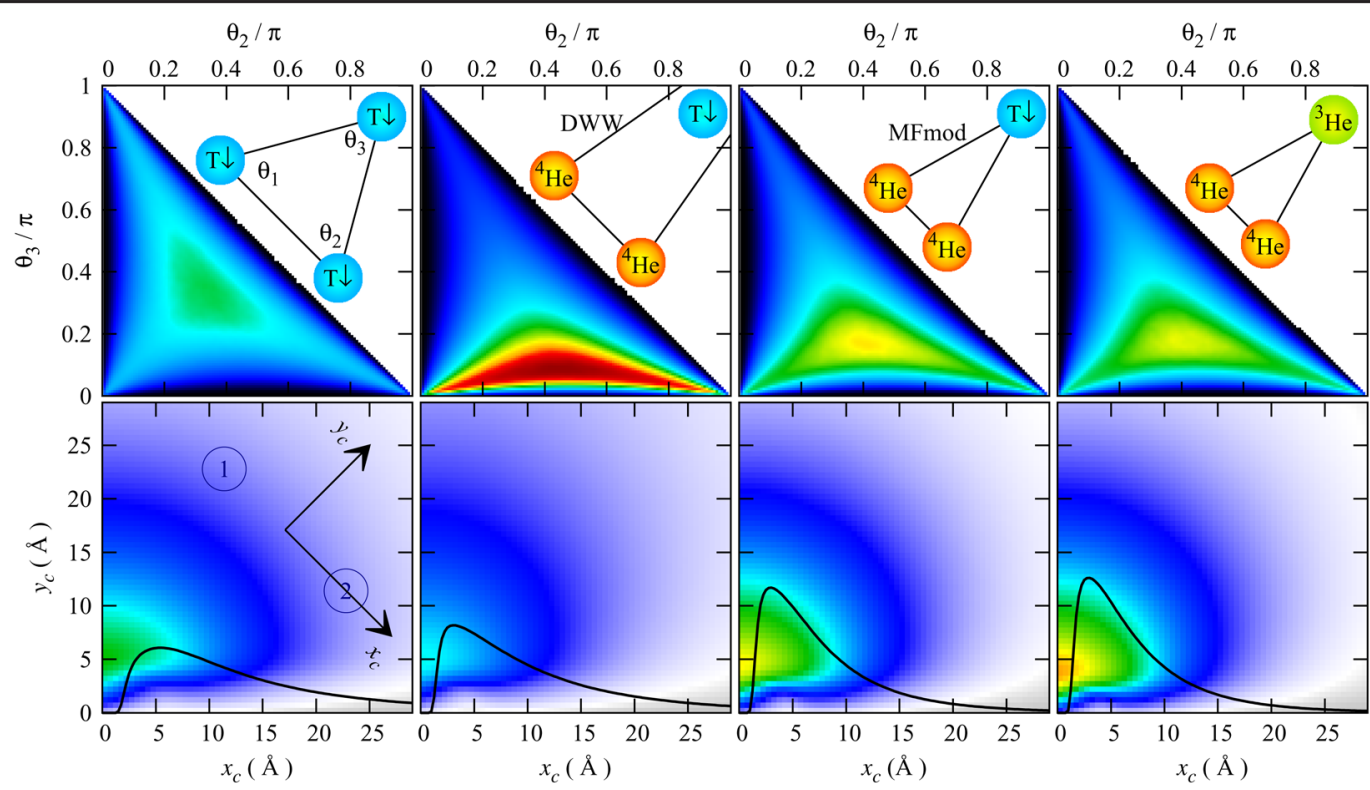

$\theta_{2} / \pi$

FIG. 3 (color online). The angular probability distribution function $P\left(\theta_{2}, \theta_{3}\right)$ in top row and spatial distributions in bottom row: of half-separations between the first and second constituent of the trimer $P_{12}\left(x_{c}\right) \equiv P\left(r_{12} / 2\right)$; and of positions of the third constituent in the plane of the trimer where $x_{c}$ starts from the center $\left(\mathbf{r}_{2}+\mathbf{r}_{1}\right) / 2$ and points in the direction of $\mathbf{r}_{2}-\mathbf{r}_{1}$.

orbits) and ${ }^{17} \mathrm{Ne}$ (core $+p+p$ ) have reduced sizes with respect to the universal line. In fact, this is expected because the universal law here obtained is constrained to $s$-wave dominated pairwise interactions, without Coulomb forces.

Let us note that molecular trimer halos appear clearly above the " $K=0$ " line in Fig. 2, where $K$ is the hypermoment. This confirms the analysis of Ref. [10], and the importance of exactly solving the Schrödinger equation.

It is worth noticing that the choice of the scaling parameter $\rho_{0}$ is not unique. Jensen et al. [47] proposed also a second definition, based on the analysis of the SQW model, where the mass $m$ is substituted by its square root $\sqrt{m}$. However, with this definition we found that the scaling appears less universal, that is systems with different masses are slightly shifted.

We also studied other structural properties of the clusters, including their shape and size. In Fig. 3, the angular and spatial probability distribution functions are shown. On the top plot, there is also a sketch of the most probable triangle structure with $\left\langle r_{12}\right\rangle$ divided by $60 \pi^{-1} \AA$. The angular probability distributions of Borromean and all bound clusters (at the edges of the plot) have the same symmetry, as expected because they are constructed from the same type of particles. However, the less bound $(\mathrm{T} \downarrow)_{3}$ is larger and more spread among different shapes. Angular distributions of the three tango states, in the middle of the plot, differ from the distributions of the Borromean or all-bound states. The distributions of ${ }^{4} \mathrm{He}_{2}{ }^{3} \mathrm{He}$ and ${ }^{4} \mathrm{He}_{2} \mathrm{~T} \downarrow$ with the modified Meyer-Frommhold (MFmod) potential [39], which are close on the universal plot, appear very similar, which is also the case if one calculates the weights of different configurations (linear, isoceles, scaline, equilateral). ${ }^{4} \mathrm{He}_{2} \mathrm{~T} \downarrow$, using the Das-Wagner-Wahl [38] potential, is more weakly bound and larger. In particular, $\mathrm{T}$ is more separated from the ${ }^{4} \mathrm{He}_{2}$ than in the case of the MFmod potential, which can be seen both from $P\left(\theta_{2}, \theta_{3}\right)$ and $P\left(x_{c}, y_{c}\right)$ distributions.

Summarizing, we studied a rather complete set of molecular halo clusters solving the Schrödinger equation in an exact way for both dimers and trimers. In the case of dimers, we identified the best scaling variables, both in energy and size, which allow for a universal line on top of which all molecular halo states stand. The analysis of the trimers is richer due to the different types of halo states one defines according to the bound or unbound pairs in which a triplet can be decomposed. For the first time, we were able to establish both the more convenient scaling variables and the universal line which trimer halo states do follow. The achievement of this universal behavior was possible due to the accuracy of the interatomic potentials used. Previous attempts of tracing this scaling law in nuclear systems were not possible due to the approximate validity of the fewbody approach and the complexity of nucleon-nucleon potentials. It is remarkable, and probably unexpected, that the universal law extends even significantly below the halo limit for both dimers and trimers. Finally, we were able to observe, and determine when, the tango universal line departs from the Borromean one as predicted by Frederico et al. [8].

L. V. M., P. S., and I. B. acknowledge support from MSES (Croatia) Grant No. 177-1770508-0493, and L. V. M. partial support from the Fulbright program. 
J. B. acknowledges partial financial support from the Ministerio de Ciencia e Innovación (MICINN) (Spain) Grant No. FIS2011-25275 and Generalitat de Catalunya Grant No. 2009SGR-1003. The computational resources of the Isabella cluster at Zagreb University Computing Center (Srce), the HYBRID cluster at the University of Split, Faculty of Science and Croatian National Grid Infrastructure (CRO NGI) were used.

*Corresponding author. leandra@pmfst.hr

[1] A. S. Jensen, K. Riisager, D. Fedorov, and E. Garrido, Rev. Mod. Phys. 76, 215 (2004).

[2] K. Riisager, Phys. Scr., T152, 014001 (2013).

[3] I. Tanihata et al., Phys. Lett. B 160, 380 (1985).

[4] I. Tanihata, H. Hamagaki, O. Hashimoto, Y. Shida, N. Yoshikawa, K. Sugimoto, O. Yamakawa, and T. Kobayashi N. Takahashi, Phys. Rev. Lett. 55, 2676 (1985).

[5] T. Frederico, A. Delfino, Lauro Tomio, and M. T. Yamashita, Prog. Part. Nucl. Phys. 67, 939 (2012).

[6] C. Chin, R. Grimm, P. Julienne, and E. Tiesinga, Rev. Mod. Phys. 82, 1225 (2010).

[7] E. Braaten and H. Hammer, Phys. Rep. 428, 259 (2006).

[8] T. Frederico, Lauro Tomio, A. Delfino, M. R. Hadizadeh, and M. T. Yamashita, Few Body Systems 51, 87 (2011).

[9] K. Riisager, D. V. Fedorov, and A. S. Jensen, Europhys. Lett. 49, 547 (2000).

[10] A. S. Jensen, K. Riisager, D. V. Fedorov, and E. Garrido, Europhys. Lett. 61, 320 (2003).

[11] P. Stipanović, L. V. Markić, J. Boronat, and B. Kežić, J. Chem. Phys. 134, 054509 (2011).

[12] D. Blume, B. D. Esry, C. H. Greene, N. N. Klausen, and G. J. Hanna, Phys. Rev. Lett. 89, 163402 (2002).

[13] M. Salci, S. B. Levin, and N. Elander, Phys. Rev. A 69, 044501 (2004).

[14] Y. Li, W. Zhang, Q. Gou, H. Song, and T. Shi, Phys. Rev. A 82, 022515 (2010).

[15] U. Kleinekathofer, M. Lewerenz, and M. Mladenovic, Phys. Rev. Lett. 83, 4717 (1999).

[16] J. Yuan and C. D. Lin, J. Phys. B 31, L637 (1998).

[17] H. Suno and B. D. Esry, Phys. Rev. A 80, 062702 (2009).

[18] Y. Li, Q. Gou, and T. Shi, Phys. Rev. A 74, 032502 (2006).

[19] I. Baccarelli, G. Delgado-Barrio, F. A. Gianturco, T. González-Lezana, S. Miret-Artes, and P. Villarreal, Phys. Chem. Chem. Phys. 2, 4067 (2000).

[20] H. Suno, E. Hiyama, and M. Kamimura, Few Body Systems 54, 1557 (2013).

[21] W. Schöllkopf and J. P. Toennies, Science 266, 1345 (1994).
[22] A. Kalinin, O. Kornilov, W. Schöllkopf, and J. P. Toennies, Phys. Rev. Lett. 95, 113402 (2005).

[23] N. Tariq, N. A. Taisan, V. Singh, and J. D. Weinstein, Phys. Rev. Lett. 110, 153201 (2013).

[24] K. Riisager, Rev. Mod. Phys. 66, 1105 (1994).

[25] R. A. Aziz, F. R. W. McCourt, and C. C. K. Wong, Mol. Phys. 61, 1487 (1987).

[26] K. T. Tang, J. P. Toennies, and C. L. Yiu, Phys. Rev. Lett. 74, 1546 (1995).

[27] T. Korona, H. L. Williams, R. Bukowski, B. Jeziorski, and K. Szalewicz, J. Chem. Phys. 106, 5109 (1997).

[28] D. Cvetko, A. Lausi, A. Morgante, F. Tommasini, P. Cortona, and M. G. Dondi, J. Chem. Phys. 100, 2052 (1994).

[29] M. Jeziorska, W. Cencek, B. Patkowski, B. Jeziorski, and K. Szalewicz, J. Chem. Phys. 127, 124303 (2007).

[30] U. Kleinekathofer, Chem. Phys. Lett. 324, 403 (2000).

[31] V. Roudnev and M. Cavagnero, J. Phys. B 45, 025101 (2012).

[32] Y. Li, H. Song, Q. Gou, H. Han, and T. Shi, Phys. Rev. A 79, 024501 (2009).

[33] Y. Li, D. Huang, Q. Gou, H. Han, and T. Shi, Phys. Rev. A 84, 014501 (2011).

[34] D. V. Fedorov, A. S. Jensen, and K. Riisager, Phys. Rev. C 49, 201 (1994).

[35] J. Boronat and J. Casulleras, Phys. Rev. B 49, 8920 (1994).

[36] J. Casulleras and J. Boronat, Phys. Rev. B 52, 3654 (1995).

[37] M. J. Jamieson, A. Dalgarno, and L. Wolniewicz, Phys. Rev. A 61, 042705 (2000).

[38] G. Das, A. F. Wagner, and A. C. Wahl, J. Chem. Phys. 68, 4917 (1978).

[39] H-K. Chung and A. Dalgarno, Phys. Rev. A 66, 012712 (2002).

[40] See Supplemental Material at http://link.aps.org/ supplemental/10.1103/PhysRevLett.113.253401 for the tables of scattering parameters, scaled energy, and scaled size of selected realistic and model trimers.

[41] R. Guardiola and J. Navarro, Phys. Rev. A 68, 055201 (2003).

[42] H. Suno, Few Body Systems 55, 229 (2014).

[43] U. Kleinekathöfer, K. T. Tang, J. P. Toennies, and C. L. Yiu, Chem. Phys. Lett. 249, 257 (1996).

[44] F. Robicheaux, Phys. Rev. A 60, 1706 (1999).

[45] M. T. Yamashita, L. Tomio, and T. Frederico, Nucl. Phys. A735, 40 (2004).

[46] I. Bešlić, L. V. Markić, and J. Boronat, J. Chem. Phys. 128, 064302 (2008).

[47] A. S. Jensen, K. Riisager, D. V. Fedorov, and E. Garrido, Europhys. Lett. 61, 320 (2003). 